\title{
Characteristics of multihole collimator gamma camera simulation modeled using MCNP5
}

\begin{abstract}
This paper describes the characteristics of the multihole collimator gamma camera that is simulated using the combination of the Monte Carlo N-Particles Code (MCNP) version 5 and in-house software. The model is constructed based on the GCA-7100A Toshiba Gamma Camera at the Royal Surrey County Hospital, Guildford, Surrey, UK. The characteristics are analyzed based on the spatial resolution of the images detected by the Sodium Iodide (NaI) detector. The result is recorded in a list-mode file referred to as a PTRAC file within MCNP5. All pertinent nuclear reaction mechanisms, such as Compton and Rayleigh scattering and photoelectric absorption are undertaken by MCNP5 for all materials encountered by each photon. The experiments were conducted on Tl-201, Co-57, Tc-99 m and Cr-51 radio nuclides. The comparison of full width half maximum value of each datasets obtained from experimental work, simulation and literature are also reported in this paper. The relationship of the simulated data is in agreement with the experimental results and data obtained in the literature. A careful inspection at each of the data points of the spatial resolution of Tc-99 $\mathrm{m}$ shows a slight discrepancy between these sets. However, the difference is very insignificant, i.e. less than $3 \mathrm{~mm}$ only, which corresponds to a size of less than 1 pixel only (of the segmented detector).
\end{abstract}

Keyword: Gamma camera; MCNP5; Multihole collimator 